But in vitro estimates ${ }^{10,11}$ of the force per myosin head give a value of $5 \mathrm{pN}$ or more. These results mean that, after allowing for filament compliance, the compliance of the crossbridge spring may be less than $2 \mathrm{~nm}$ per $5 \mathrm{pN}$. As the spring compliance is reduced, so is the distance it can be stretched by thermal energy, which is a key parameter in conventional cross- bridge theories of muscle contraction. The new mechanical and structural nanotechnologies may soon provide a definitive test of these theories.

Malcolm Irving is at The Randall Institute, Division of Biomedical Sciences, King's College London, 26-29 Drury Lane, London WC2B 5RL, UK

\title{
Mixed predictions
}

\section{R. P. Behringer}

AN understanding of the dynamics of granular materials is extremely important for a host of technical processes ranging from the seemingly mundane storage and retrieval of coal in silos to the high-tech processing of pharmaceutical powders ${ }^{1}$. As yet, our understanding sadly lags that for other materials ${ }^{2,3}$. But on page 39 of this issue, Metcalfe et al. ${ }^{4}$ demonstrate that a simple but original model can describe a good deal about the particularly important process of mixing.

Granular materials can be thought of as an intermediate phase of matter: they can sustain shear like a solid - up to a point but they can also flow like water. Once flow begins, the motion can contain a large random component, which is sometimes described in terms of a granular temperature. Yet energy, random or otherwise, is rapidly dissipated for granular flows. And, in many cases, static regions adjoin flowing regions. A conventional thermodynamic or hydrodynamic description is not appropriate. So what Metcalfe et al. have done is to combine experiments with a simple but novel model for mixing of a homogeneous material. By contrast, much other work has focused on the segregation that occurs for mixtures of particles of different sizes ${ }^{5-7}$. A key feature of the work by Metcalfe $e t$ $a l$. is that it cleanly separates deterministic and random aspects of mixing. From this work comes a specific prediction for optimum mixing efficiency.

Metcalfe et al. consider an idealized drum mixer which is thin enough that the motion is effectively two-dimensional. The axis of the drum is horizontal, and as it rotates, the upper surface of the material inside becomes unstable to avalanches. The rotation rate is slow, so that each avalanche stops before the next begins. The instability angle is $\theta_{i}$, and after an avalanche, the surface of the material relaxes to one with a lower slope, $\theta_{\mathrm{f}}<\theta_{\mathrm{i}}$. The effect of an avalanche is to transfer a thin wedge of material from the upper to the lower part of the surface. Once material has fallen to the lower side of the incline, it remains there until rotation of the drum brings the material back to the top once more.

Metcalfe et al. find experimentally a very complex process as the grains avalanche down the upper surface, and an exactly describable rotation thereafter, until the material once again is ready to avalanche. If the drum is exactly half full, a wedge will avalanche and rotate repeatedly without any mixing between wedges. But by simple geometry, if the drum is less than half full, a wedge of material from a given avalanche will not stay in the same wedge on successive avalanches: different wedges will mix. This is a purely geometric process, as sketched in the figure. In each case, position 1 corresponds to the point at which a wedge avalanches. After the avalanche, the wedge is at position 2 . Position 3 corresponds to a later time when the wedge has rotated part way around. For the first case, nothing will have happened to the wedge in question. But for the second case, the tip of the wedge will already have been mixed with other material at point 3. Mixing between wedges also occurs if the drum is more than half full, so that $f=1 / 2$ is a particularly inefficient filling fraction for mixing. If

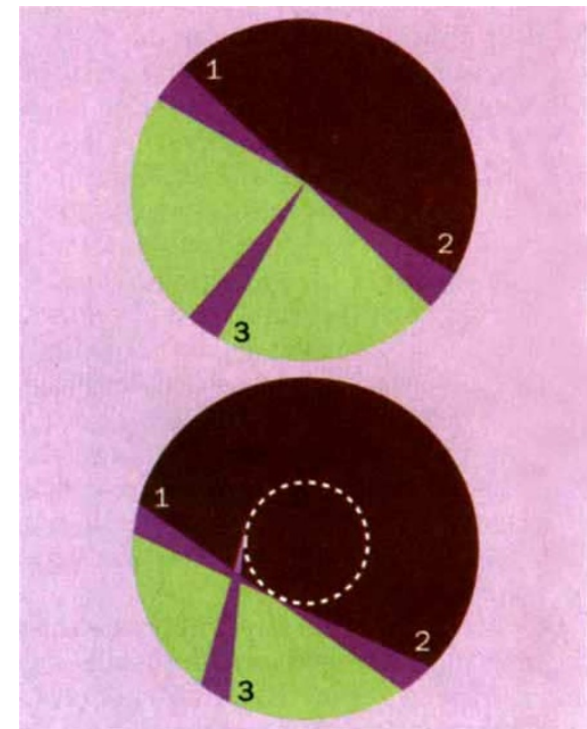

Mixing 'wedges' of granular material. Top, for a half-filled drum of grains; bottom, for a drum that is less than half full. one characterizes the mixing by $\gamma$, the rate at which material within the container becomes homogenized, this rate is largest for small filling fraction $f$; falls to zero (no mixing) for $f=1 / 2$; rises somewhat for $f$ increasing above $1 / 2$ and is again zero for a full container, $f=1$.

The details of how mixing occurs during an avalanche are unclear. In their model, Metcalfe et al. assume that this is a random process. They then incorporate the geometric effect of wedges as described above, which is totally deterministic.

Although $\gamma$ is an important measure of mixing, a practical concern is to produce as large a volume of mixed material as possible in a given time. If $V$ represents the fractional volume of the drum which is occupied by material, then one measure of the efficiency is $\chi=\gamma V$. Metcalfe et al. predict a maximum for $\chi$ when $f=0.25$, and they obtain a maximum efficiency in their experiments when $f=0.23$. This is excellent agreement.

Important questions remain. Perhaps the most interesting and technically relevant of these is what happens if the grains have a range of sizes. This work shows that the important additional feature to describe this process will be the dynamics during the avalanche. For instance, if one size of particle consistently tends to avalanche closer to the curved wall of the drum, there will be radial segregation by size, as in the recent experiments of Clément et al. ${ }^{8}$. In order to capture such features, the model must account for each grain size separately. In the simplest case of two distinct grain sizes, the geometric part of the model would clearly need modification to account for the fact that, after the avalanche, the particles of a given size would no longer be distributed uniformly in the wedge. But this avalanche-induced segregation is unlikely to be perfect, and thus the random part of the model would also need to be modified. Additional issues that will need to be addressed include three-dimensional effects, and mixing in non-cylindrical geometries. Although the mathematical analysis may be more complicated in these cases, the key idea of separating geometric and random mixing will still apply.

R. $P$. Behringer is in the Department of Physics, Duke University, Durham, North Carolina 27708, USA.

1. Merrow, E. W. A Quantitative Assessment of R\&D Requirements for Solids Processing Technology (Rand Corp. Report R-3216-DOE/PSSP, 1986).

2. Jaeger, H. M. \& Nagel, S. R. Science 255, 1523 (1992) 3. Behringer, R. P. Nonlinear Sci. Today 3, 1 (1993)

4. Metcalfe, G., Shinbrot, T., McCarthy, J. J. \& Ottino, J. M. Nature 374, 39-41 (1995)

5. Rosato, A. et al. Phys. Rev. Lett. 58, 1038-1040 (1987). 6. Jullien, R. \& Meakin, P. Nature 344, 425-427 (1990)

7. Knight, J. B., Jaeger, H. M. \& Nagel, S. R. Phys. Rev. Lett. 70,3728-3731 (1993).

8. Clément, E., Duran, J. \& Rajchenbach, J. in C. R. 11 Congr. Soc. Fr. méchanique 4, 327 (1993). 